\title{
COMPLETE HOMOMORPHISMS BETWEEN MODULE LATTICES
}

\author{
Patrick F. Smith \\ Received: 18 October 2013; Revised: 25 May 2014 \\ Communicated by Christian Lomp \\ For my good friend John Clark on his 70th birthday
}

\begin{abstract}
We examine the properties of certain mappings between the lattice $\mathcal{L}(R)$ of ideals of a commutative ring $R$ and the lattice $\mathcal{L}\left({ }_{R} M\right)$ of submodules of an $R$-module $M$, in particular considering when these mappings are complete homomorphisms of the lattices. We prove that the mapping $\lambda$ from $\mathcal{L}(R)$ to $\mathcal{L}\left({ }_{R} M\right)$ defined by $\lambda(B)=B M$ for every ideal $B$ of $R$ is a complete homomorphism if $M$ is a faithful multiplication module. A ring $R$ is semiperfect (respectively, a finite direct sum of chain rings) if and only if this mapping $\lambda: \mathcal{L}(R) \rightarrow \mathcal{L}\left({ }_{R} M\right)$ is a complete homomorphism for every simple (respectively, cyclic) $R$-module $M$. A Noetherian ring $R$ is an Artinian principal ideal ring if and only if, for every $R$-module $M$, the mapping $\lambda: \mathcal{L}(R) \rightarrow \mathcal{L}\left({ }_{R} M\right)$ is a complete homomorphism.
\end{abstract}

Mathematics Subject Classification 2010: 06B23, 06B10, 16D10, 16D80

Keywords: Lattice of ideals, lattice of submodules, multiplication modules, complete lattice, complete homomorphism

\section{Introduction}

In this paper we continue the discussion in [7] concerning mappings, in particular homomorphisms, between the lattice of ideals of a commutative ring and the lattice of submodules of a module over that ring.

A lattice $L$ is called complete provided every non-empty subset $S$ has a least upper bound $\vee S$ and a greatest lower bound $\wedge S$. Given complete lattices $L$ and $L^{\prime}$ we say that a mapping $\varphi: L \rightarrow L^{\prime}$ is a complete homomorphism provided

$$
\varphi(\vee S)=\vee\{\varphi(x): x \in S\} \text { and } \varphi(\wedge S)=\wedge\{\varphi(x): x \in S\},
$$

for every non-empty subset $S$ of $L$. A complete homomorphism which is a bijection (respectively, injection, surjection) will be called a complete isomorphism (respectively, complete monomorphism, complete epimorphism). The first result is standard and easy to prove. 
Lemma 1.1. The following statements are equivalent for a bijection $\varphi$ from a complete lattice $L$ to a complete lattice $L^{\prime}$.

(i) $\varphi$ is a complete isomorphism.

(ii) $\varphi(\vee S)=\vee\{\varphi(x): x \in S\}$ for every non-empty subset $S$ of $L$.

(iii) $\varphi(\wedge S)=\wedge\{\varphi(x): x \in S\}$ for every non-empty subset $S$ of $L$.

Moreover, in this case the inverse mapping $\varphi^{-1}: L^{\prime} \rightarrow L$ is also a complete isomorphism.

An element $x$ of a complete lattice $L$ is called compact in case whenever $x \leq \vee S$, for some non-empty subset $S$ of $L$, there exists a finite subset $F$ of $S$ such that $x \leq \vee F$. The next result is also easy to prove.

Lemma 1.2. Let $\varphi: L \rightarrow L^{\prime}$ be a complete isomorphism from a complete lattice $L$ to a complete lattice $L^{\prime}$ and let $x$ be a compact element of $L$. Then $\varphi(x)$ is a compact element of $L^{\prime}$.

A lattice $L$ is called distributive in case

$$
x \wedge(y \vee z)=(x \wedge y) \vee(x \wedge z)
$$

for all elements $x, y, z$ in $L$. The next result is also well known and easy to prove. It states that a lattice is distributive if and only if its dual lattice is distributive.

Lemma 1.3. A lattice $L$ is distributive if and only if $x \vee(y \wedge z)=(x \vee y) \wedge(x \vee z)$ for all $x, y, z$ in $L$.

Throughout this note all rings will be commutative with identity and all modules will be unital. Let $R$ be a ring and $M$ be any $R$-module. Let $\mathcal{L}(R)$ denote the lattice of all ideals of the ring $R$ and let $\mathcal{L}\left({ }_{R} M\right)$ denote the lattice of all submodules of the $R$-module $M$. In [7] we investigate the mapping $\lambda: \mathcal{L}(R) \rightarrow \mathcal{L}\left({ }_{R} M\right)$ defined by $\lambda(B)=B M$ for every ideal $B$ of $R$ and the mapping $\mu: \mathcal{L}\left({ }_{R} M\right) \rightarrow \mathcal{L}(R)$ defined by $\mu(N)=\left(N:_{R} M\right)$ for every submodule $N$ of $M$, where $\left(N:_{R} M\right)$ denotes the set of elements $r \in R$ such that $r M \subseteq N$. The module $M$ is called a $\lambda$-module in [7] in case $\lambda: \mathcal{L}(R) \rightarrow \mathcal{L}\left({ }_{R} M\right)$ is a homomorphism. Similarly, in [7] the module $M$ is called a $\mu$-module if the above mapping $\mu$ is a homomorphism. For any unexplained terminology and notation, please see [7].

Note that the lattice $\mathcal{L}\left({ }_{R} M\right)$ is complete when we define

$$
\wedge \mathcal{S}=\cap_{N \in \mathcal{S}} N \text { and } \vee \mathcal{S}=\sum_{N \in \mathcal{S}} N
$$


for every non-empty collection $\mathcal{S}$ of submodules of $M$. In particular the lattice $\mathcal{L}(R)$ is complete. The module $M$ will be called $\lambda$-complete in case the above mapping $\lambda: \mathcal{L}(R) \rightarrow \mathcal{L}\left({ }_{R} M\right)$ is a complete homomorphism. Similarly the module $M$ will be called $\mu$-complete if $\mu: \mathcal{L}\left({ }_{R} M\right) \rightarrow \mathcal{L}(R)$ is a complete homomorphism. It is clear that every $\lambda$-complete module is a $\lambda$-module and every $\mu$-complete module is a $\mu$-module but, in each case, the converse is false in general, as we can easily show.

For example, let $\mathbb{Z}$ denote the ring of rational integers and let $p$ be any prime in $\mathbb{Z}$. Then the simple $\mathbb{Z}$-module $U=\mathbb{Z} / \mathbb{Z} p$ is a $\lambda$-module. Let $q$ be any prime in $\mathbb{Z}$ other than $p$ and let $\mathcal{S}$ denote the collection of ideals of $\mathbb{Z}$ of the form $\mathbb{Z} q^{n}$ for all positive integers $n$. Then

$$
\lambda(\wedge \mathcal{S})=\lambda\left(\cap_{n \geq 1} \mathbb{Z} q^{n}\right)=\lambda(0)=0,
$$

but

$$
\wedge\{\lambda(B): B \in \mathcal{S}\}=\cap_{n \geq 1} q^{n} U=U .
$$

Thus $U$ is not $\lambda$-complete.

Now let $\mathbb{Z}\left(p^{\infty}\right)$ denote the Prüfer $p$-group for any prime $p$ in $\mathbb{Z}$. Let $V=\mathbb{Z}\left(p^{\infty}\right)$. Then the $\mathbb{Z}$-module $V$ is a $\mu$-module (see [7, Example 3.11]). However $V$ contains an infinite collection $\mathcal{T}$ of proper submodules $V_{i}(i \in I)$ such that $V=\cup_{i \in I} V_{i}$. Thus

$$
\mu(\vee \mathcal{T})=\mu(V)=(V: \mathbb{Z} V)=\mathbb{Z},
$$

but

$$
\vee\{\mu(W): W \in \mathcal{T}\}=\sum_{i \in I} \mu\left(V_{i}\right)=\sum_{i \in I}\left(V_{i}: \mathbb{Z} V\right)=0 .
$$

Thus the $\mathbb{Z}$-module $V$ is not $\mu$-complete.

Proposition 1.4. Given any ring $R$ and $R$-module $M$ the following statements are equivalent.

(i) The mapping $\lambda: \mathcal{L}(R) \rightarrow \mathcal{L}\left({ }_{R} M\right)$ is a complete isomorphism.

(ii) The mapping $\mu: \mathcal{L}\left({ }_{R} M\right) \rightarrow \mathcal{L}(R)$ is a complete isomorphism.

Moreover, in this case $M$ is a faithful $R$-module.

Proof. (i) $\Leftrightarrow$ (ii) By Lemma 1.1 and [7, Corollary 1.5].

Now suppose that (i) holds. Let $A=\operatorname{ann}_{R}(M)$. Then $\lambda(A)=A M=0=0 M=$ $\lambda(0)$ so that $A=0$ and $M$ is faithful.

Again let $R$ be a ring and let $M$ be an $R$-module. Let $A=\operatorname{ann}_{R}(M)$. By defining

$$
(r+A) m=r m \quad(r \in R, m \in M),
$$


$M$ becomes a faithful $(R / A)$-module with the property that a subset $X$ of $M$ is an $R$-submodule of $M$ if and only if $X$ is an $(R / A)$-submodule of $M$. Thus the lattice $\mathcal{L}\left({ }_{R} M\right)$ is identical to the lattice $\mathcal{L}\left({ }_{R / A} M\right)$. The mapping $\lambda: \mathcal{L}(R / A) \rightarrow \mathcal{L}\left({ }_{R / A} M\right)$ will be denoted by $\bar{\lambda}$. Note that if $\bar{B}$ is any ideal of the $\operatorname{ring} R / A$ then $\bar{B}=B / A$ for a unique ideal $B$ of $R$ containing $A$ and hence

$$
\bar{\lambda}(\bar{B})=\bar{\lambda}(B / A)=(B / A) M=B M .
$$

In addition, the mapping $\mu: \mathcal{L}\left({ }_{R / A} M\right) \rightarrow \mathcal{L}(R / A)$ is denoted by $\bar{\mu}$ so that

$$
\bar{\mu}(N)=\left(N:_{R / A} M\right)=\left(N:_{R} M\right) / A,
$$

for every submodule $N$ of $M$, noting that, of course, $A \subseteq\left(N:_{R} M\right)$ for every submodule $N$ of $M$.

Let $R$ be any ring. An $R$-module $M$ is called a multiplication module in case for each submodule $N$ of $M$ there exists an ideal $B$ of $R$ such that $N=B M$. Cyclic modules are multiplication modules as are projective ideals of $R$ or ideals of $R$ generated by idempotent elements (see [2]). We prove that for any ring $R$ an $R$-module $M$ is $\mu$-complete if and only if $M$ is a finitely generated multiplication module (Theorem 2.2). An easy consequence is that the mapping $\mu$ (respectively, $\lambda$ ) is a complete isomorphism if and only if $M$ is a finitely generated faithful multiplication module (Corollary 2.4).

For any ring $R$, projective modules are $\lambda$-complete (Corollary 3.4) as are faithful multiplication modules (Theorem 3.6). We prove that a ring $R$ is arithmetical if and only if every $R$-module is a $\lambda$-module (Theorem 4.6). The ring $R$ is semiperfect if and only if every simple $R$-module is $\lambda$-complete (Theorem 4.2). On the other hand, $R$ is a direct sum of chain rings if and only if every cyclic $R$-module $M$ is $\lambda$-complete (Theorem 4.7). Note that we do not yet know which rings $R$ have the property that every $R$-module is $\lambda$-complete. It is proved that a Noetherian ring $R$ is an Artinian principal ideal ring if and only if every $R$-module is $\lambda$-complete (Theorem 4.12).

\section{2. $\mu$-complete modules}

Let $R$ be a ring and let $M$ be an $R$-module. In this section we shall investigate $\mu$-complete modules. We begin with the following basic result.

Lemma 2.1. Given any ring $R$, an $R$-module $M$ is $\mu$-complete if and only if $\left(\sum_{N \in \mathcal{T}} N:_{R} M\right)=\sum_{N \in \mathcal{T}}\left(N:_{R} M\right)$ for any non-empty collection $\mathcal{T}$ of submodules of $M$. 
Proof. Let $\mathcal{T}$ be any non-empty collection of submodules of $M$. Then

$$
\begin{gathered}
\mu(\wedge \mathcal{T})=\mu\left(\cap_{N \in \mathcal{T}} N\right)=\left(\cap_{N \in \mathcal{T}} N:_{R} M\right)=\cap_{N \in \mathcal{T}}\left(N:_{R} M\right) \\
=\wedge\{\mu(N): N \in \mathcal{T}\} .
\end{gathered}
$$

On the other hand

$$
\mu(\vee \mathcal{T})=\mu\left(\sum_{N \in \mathcal{T}} N\right)=\left(\sum_{N \in \mathcal{T}} N:_{R} M\right)
$$

and

$$
\vee\{\mu(N): N \in \mathcal{T}\}=\sum_{N \in \mathcal{T}}\left(N:_{R} M\right)
$$

The result follows.

Note that, given any $\operatorname{ring} R$ and $R$-module $M$, the mapping $\mu$ is not a surjection in case $M$ is not a faithful $R$-module because in this case no submodule $N$ of $M$ has the property that $\left(N:_{R} M\right)=0$. The next result characterizes $\mu$-complete modules.

Theorem 2.2. Given any ring $R$, the following statements are equivalent for an $R$-module $M$ with annihilator $A$ in $R$.

(i) $M$ is $\mu$-complete.

(ii) $M$ is a finitely generated multiplication module.

(iii) The mapping $\bar{\mu}: \mathcal{L}\left({ }_{R / A} M\right) \rightarrow \mathcal{L}(R / A)$ is a complete isomorphism.

(iv) The mapping $\bar{\lambda}: \mathcal{L}(R / A) \rightarrow \mathcal{L}\left({ }_{R / A} M\right)$ is a complete isomorphism.

Moreover in this case the mapping $\mu: \mathcal{L}\left({ }_{R} M\right) \rightarrow \mathcal{L}(R)$ is a monomorphism.

Proof. (i) $\Rightarrow$ (ii) Let $\mathcal{T}$ denote the collection of all cyclic submodules of the $\mu$ complete module $M$. Then $M=\sum_{N \in \mathcal{T}} N$. By Lemma 2.1,

$$
R=\left(M:_{R} M\right)=\left(\sum_{N \in \mathcal{T}} N:_{R} M\right)=\sum_{N \in \mathcal{T}}\left(N:_{R} M\right),
$$

and hence $R=\left(R m_{1}:_{R} M\right)+\cdots+\left(R m_{n}:_{R} M\right)$ for some positive integer $n$ and elements $m_{i} \in M(1 \leq i \leq n)$. It follows that

$$
M=R M=\left(R m_{1}:_{R} M\right) M+\cdots+\left(R m_{n}:_{R} M\right) M \subseteq R m_{1}+\cdots+R m_{n} \subseteq M .
$$

Therefore $M=R m_{1}+\cdots+R m_{n}$. In other words, $M$ is finitely generated. By [7, Theorem 3.8], $M$ is also a multiplication module.

(ii) $\Rightarrow$ (i) Suppose that $M$ is a finitely generated multiplication module. By [7, Lemma 3.1 and Theorem 3.8] and induction,

$$
\left(K_{1}+\cdots+K_{n}:_{R} M\right)=\left(K_{1}:_{R} M\right)+\cdots+\left(K_{n}:_{R} M\right),
$$


for every positive integer $n$ and submodules $K_{i}(1 \leq i \leq n)$. Let $L_{i}(i \in I)$ be any non-empty collection of submodules of $M$. Clearly,

$$
\sum_{i \in I}\left(L_{i}:_{R} M\right) \subseteq\left(\sum_{i \in I} L_{i}:_{R} M\right) .
$$

Let $r \in\left(\sum_{i \in I} L_{i}:_{R} M\right)$. Then $r M$ is a finitely generated submodule of $\sum_{i \in I} L_{i}$. There exists a finite subset $I^{\prime}$ of $I$ such that $r M \subseteq \sum_{i \in I^{\prime}} L_{i}$. Hence

$$
r \in\left(\sum_{i \in I^{\prime}} L_{i}:_{R} M\right)=\sum_{i \in I^{\prime}}\left(L_{i}:_{R} M\right) \subseteq \sum_{i \in I}\left(L_{i}:_{R} M\right) .
$$

Thus $\left(\sum_{i \in I} L_{i}:_{R} M\right) \subseteq \sum_{i \in I}\left(L_{i}:_{R} M\right)$ and we have proved that $\left(\sum_{i \in I} L_{i}:_{R}\right.$ $M)=\sum_{i \in I}\left(L_{i}:_{R} M\right)$. By Lemma $2.1, M$ is $\mu$-complete.

(ii) $\Rightarrow$ (iii) By [7, Lemma 2.9], the $(R / A)$-module $M$ is a finitely generated faithful multiplication module and hence the mapping $\bar{\mu}$ is a bijection by [7, Theorem 4.3]. By the proof of (ii) $\Rightarrow$ (i), the mapping $\bar{\mu}$ is a complete isomorphism.

(iii) $\Leftrightarrow$ (iv) By Proposition 1.4.

(iii) $\Rightarrow$ (ii) By the proof of (i) $\Rightarrow$ (ii), the $(R / A)$-module $M$ is a finitely generated multiplication module and hence the $R$-module $M$ is a finitely generated multiplication module by [7, Lemma 2.9].

Finally, suppose that there exist submodules $N$ and $L$ of $M$ such that $\mu(N)=$ $\mu(L)$. By [2, p. 756],

$$
N=\left(N:_{R} M\right) M=\mu(N) M=\mu(L) M=\left(L:_{R} M\right) M=L .
$$

Thus $\mu$ is a monomorphism.

Given a ring $R$ and an $R$-module $M$, note that Theorem 2.2 shows that whenever the mapping $\mu: \mathcal{L}\left({ }_{R} M\right) \rightarrow \mathcal{L}(R)$ is a complete homomorphism then it is a monomorphism. This is not true if $\mu$ is merely a homomorphism (see, for example, [7, Example 3.11 and Proposition 3.12]).

Corollary 2.3. Every homomorphic image of a $\mu$-complete module $M$ is $\mu$-complete.

Proof. By Theorem 2.2.

In contrast to Corollary 2.3 homomorphic images of $\lambda$-complete modules need not be $\lambda$-complete. For example, the $\mathbb{Z}$-module $\mathbb{Z}$ is $\lambda$-complete but we have already noted that the simple $\mathbb{Z}$-module $\mathbb{Z} / \mathbb{Z} p$ is not $\lambda$-complete for every prime $p$ in $\mathbb{Z}$. (Note that every homomorphic image of a $\lambda$-module over the $\operatorname{ring} \mathbb{Z}$ is also a $\lambda$ module by [7, Theorem 2.3].)

Corollary 2.4. Given a ring $R$, the following statements are equivalent for an $R$-module $M$. 
(i) The mapping $\lambda: \mathcal{L}(R) \rightarrow \mathcal{L}\left({ }_{R} M\right)$ is a complete isomorphism.

(ii) The mapping $\mu: \mathcal{L}\left({ }_{R} M\right) \rightarrow \mathcal{L}(R)$ is a complete isomorphism.

(iii) The R-module $M$ is a finitely generated faithful multiplication module.

Proof. By Proposition 1.4 and Theorem 2.2.

Corollary 2.5. Let $R$ be a ring and let $M$ be any $\mu$-complete $R$-module with $A=$ ann $_{R}(M)$. Then the $(R / A)$-module $M$ is a $\lambda$-complete module.

Proof. By [7, Lemma 2.9], Theorem 2.2 and Corollary 2.4.

Note that in general $\mu$-complete modules are not $\lambda$-complete. For, let $R$ be a domain that is not Prüfer. By [7, Theorem 2.3], there exists a cyclic $R$-module $M$ which is not a $\lambda$-module and hence is not $\lambda$-complete. However, every cyclic module over any ring is a finitely generated multiplication module.

\section{3. $\lambda$-complete modules}

In contrast to the case of $\mu$-complete modules, the situation for (non-faithful) $\lambda$-complete modules is more complex. We already know that simple modules over $\mathbb{Z}$ are not $\lambda$-complete although they are clearly finitely generated multiplication modules. First we prove an elementary result characterizing $\lambda$-complete modules.

Lemma 3.1. Let $R$ be a ring. Then an $R$-module $M$ is $\lambda$-complete if and only if $\left(\cap_{B \in \mathcal{S}} B\right) M=\cap_{B \in \mathcal{S}}(B M)$ for every non-empty collection $\mathcal{S}$ of ideals of $R$.

Proof. Let $\mathcal{S}$ be any non-empty collection of ideals of $R$. Then

$$
\lambda(\vee \mathcal{S})=\left(\sum_{B \in \mathcal{S}} B\right) M=\sum_{B \in \mathcal{S}}(B M)=\vee\{\lambda(B): B \in \mathcal{S}\}
$$

In addition, $\lambda(\wedge \mathcal{S})=\left(\cap_{B \in \mathcal{S}} B\right) M$ and $\wedge\{\lambda(B): B \in \mathcal{S}\}=\cap_{B \in \mathcal{S}}(B M)$. The result follows.

Corollary 3.2. Let $A$ be any ideal of a ring $R$. Then the $R$-module $R / A$ is $\lambda$ complete if and only if $\cap_{B \in \mathcal{S}}(A+B)=A+\left(\cap_{B \in \mathcal{S}} B\right)$ for every non-empty collection $\mathcal{S}$ of ideals of $R$.

Proof. Apply Lemma 3.1 to the module $M=R / A$.

Lemma 3.3. Let $R$ be any ring. Then

(a) Every direct summand of a $\lambda$-complete module is $\lambda$-complete.

(b) Every direct sum of $\lambda$-complete modules is also $\lambda$-complete. 
Proof. (a) Let $K$ be a direct summand of a $\lambda$-complete module $M$. Let $\mathcal{S}$ be any non-empty collection of ideals of $R$. Then

$$
\begin{gathered}
\left(\cap_{B \in \mathcal{S}} B\right) K=K \cap\left(\cap_{B \in \mathcal{S}} B\right) M=K \cap\left(\cap_{B \in \mathcal{S}}(B M)\right) \\
=\cap_{B \in \mathcal{S}}(K \cap B M)=\cap_{B \in \mathcal{S}}(B K) .
\end{gathered}
$$

By Lemma $3.1 K$ is a $\lambda$-complete module.

(b) Let $L_{i}(i \in I)$ be any collection of $\lambda$-complete modules and let $L=\oplus_{i \in I} L_{i}$.

Given any non-empty collection $\mathcal{S}$ of ideals of $R$ we have:

$$
\left(\cap_{B \in \mathcal{S}} B\right) L=\oplus_{i \in I}\left(\cap_{B \in \mathcal{S}} B\right) L_{i}=\oplus_{i \in I}\left(\cap_{B \in \mathcal{S}}\left(B L_{i}\right)\right)=\cap_{B \in \mathcal{S}}(B L) .
$$

By Lemma $3.1 L$ is $\lambda$-complete.

Corollary 3.4. Given any ring $R$, every projective $R$-module is $\lambda$-complete.

Proof. Clearly the $R$-module $R$ is $\lambda$-complete. Apply Lemma 3.3.

Recall the following result (see [2, Theorem 1.2] or [7, Lemma 2.10]).

Lemma 3.5. Let $R$ be any ring. Then an $R$-module $M$ is a multiplication module if and only if for each maximal ideal $P$ of $R$ either

(a) for each $m$ in $M$ there exists $p$ in $P$ such that $(1-p) m=0$, or

(b) there exist $x \in M$ and $q \in P$ such that $(1-q) M \subseteq R x$.

We now strengthen [7, Theorem 2.12].

Theorem 3.6. Let $R$ be any ring. Then every faithful multiplication $R$-module is a $\lambda$-complete module.

Proof. Let $M$ be a faithful multiplication $R$-module. Let $\mathcal{S}$ be any non-empty collection of ideals of $R$. Then $\left(\cap_{B \in \mathcal{S}} B\right) M \subseteq \cap_{B \in \mathcal{S}}(B M)$. Suppose that there exists $m \in \cap_{B \in \mathcal{S}}(B M)$ with $m \notin\left(\cap_{B \in \mathcal{S}} B\right) M$. Let $I=\left\{r \in R: r m \in\left(\cap_{B \in \mathcal{S}} B\right) M\right\}$. Then $I$ is a proper ideal of $R$. Let $P$ be a maximal ideal of $R$ such that $I \subseteq P$. Clearly $(1-p) m=0$ for some $p \in P$ implies that $1-p \in I$, a contradiction. By Lemma 3.5 there exist $x \in M$ and $q \in P$ such that $(1-q) M \subseteq R x$. Note that for each ideal $B$ in $\mathcal{S}(1-q) m \in(1-q) B M=B(1-q) M \subseteq B x$. Thus $(1-q) m=r_{B} x$ for some $r_{B} \in B$ for each ideal $B$ in $\mathcal{S}$. If $B$ and $C$ are ideals in $\mathcal{S}$ then $\left(r_{B}-r_{C}\right) x=0$ and hence $(1-q)\left(r_{B}-r_{C}\right) M=\left(r_{B}-r_{C}\right)(1-q) M \subseteq\left(r_{B}-r_{C}\right) R x=0$. Because $M$ is faithful we have $(1-q)\left(r_{B}-r_{C}\right)=0$ and $(1-q) r_{B}=(1-q) r_{C}$. It follows that $(1-q) r_{C} \in \cap_{B \in \mathcal{S}} B$. Thus $(1-q)^{2} m=(1-q) r_{C} x \in\left(\cap_{B \in \mathcal{S}} B\right) M$. This implies that $(1-q)^{2} \in I \subseteq P$, a contradiction. Thus $\cap_{B \in \mathcal{S}}(B M)=\left(\cap_{B \in \mathcal{S}} B\right) M$ for every non-empty subset $\mathcal{S}$ of ideals of $R$. By Lemma $3.1 M$ is $\lambda$-complete. 
We have already noted that for any prime $p$ in $\mathbb{Z}$, the simple $\mathbb{Z}$-module $\mathbb{Z} / \mathbb{Z} p$ is a multiplication module which is not $\lambda$-complete. Thus Theorem 3.6 requires that the module be faithful as well as a multiplication module.

If $R$ is any ring and $M$ the free $R$-module $R \oplus R$, then it is not hard to check that the mapping $\lambda: \mathcal{L}(R) \rightarrow \mathcal{L}\left({ }_{R} M\right)$ is a complete monomorphism which is not an epimorphism. On the other hand, compare the following result with Theorem 2.2 .

Proposition 3.7. Let $R$ be a ring and let $I$ be a proper ideal of $R$ which is generated by idempotent elements such that ann $n_{R}(I)=0$. Then the $R$-module $I$ is a faithful multiplication module and the mapping $\lambda: \mathcal{L}(R) \rightarrow \mathcal{L}\left({ }_{R} I\right)$ is a complete epimorphism but not a monomorphism.

Proof. By [7, Proposition 2.15] and Theorem 3.6.

\section{Special rings}

Let $R$ be any ring. Then every cyclic $R$-module is $\mu$-complete by Theorem 2.2 . However, the same theorem shows that the 2-generated $R$-module $M=R \oplus R$ is not $\mu$-complete because $M$ is not a multiplication module. Thus no non-zero ring $R$ has the property that every finitely generated $R$-module is $\mu$-complete. We saw in Corollary 3.4 that for every ring $R$ every projective $R$-module is $\lambda$-complete. In addition for every ring $R$, every faithful multiplication module is $\lambda$-complete by Theorem 3.6. In this section we investigate rings $R$ with the property that every module in a certain class of $R$-modules is $\lambda$-complete. The classes that we shall look at are the classes of simple $R$-modules, semisimple $R$-modules, cyclic $R$-modules, finitely generated $R$-modules and all $R$-modules.

First we investigate when simple modules are $\lambda$-complete. Following [1, p. 303] we call a ring $R$ with Jacobson radical $J$ a semiperfect ring in case $R / J$ is semiprime Artinian and idempotents lift modulo $J$. For properties of semiperfect rings see [1, Theorem 27.6] or [10, Theorem 42.6]. By a local ring we mean any (commutative) ring which contains only one maximal ideal. It is well known that a (commutative) ring $R$ is semiperfect if and only if $R$ is the (finite) direct sum of local rings (see, for example, [1, Theorem 27.6]). Given any $\operatorname{ring} R$, a submodule $N$ of an $R$-module $M$ has a supplement $K$ in case $K$ is a submodule of $M$ minimal with respect to the property that $M=N+K$.

Lemma 4.1. Let $R$ be a ring and let $U$ be a simple $R$-module with annihilator $P$. Then the $R$-module $U$ is $\lambda$-complete if and only if $P$ has a supplement in ${ }_{R} R$. 
Proof. Suppose first that $U$ is $\lambda$-complete. Let $\mathcal{S}$ denote the collection of ideals $B$ of $R$ such that $R=P+B$. By Corollary 3.2 $R=P+C$ where $C=\cap_{B \in \mathcal{S}} B$. Clearly $C$ is a supplement of $P$ in ${ }_{R} R$. Conversely, suppose that $P$ has a supplement $G$ in ${ }_{R} R$. Let $\mathcal{T}$ be any non-empty collection of ideals of $R$. Then

$$
P+\left(\cap_{D \in \mathcal{T}} D\right)=P=\cap_{D \in \mathcal{T}}(P+D),
$$

unless $D \nsubseteq P$ for all $D \in \mathcal{T}$. Now suppose that $D \nsubseteq P$ for all $D \in \mathcal{T}$. Let $D \in \mathcal{T}$. Then $R=P+G=P+D$ implies that $R=P+(D \cap G)$ and hence $G=D \cap G \subseteq D$. It follows that

$$
R=P+G \subseteq P+\left(\cap_{D \in \mathcal{T}} D\right) \subseteq \cap_{D \in \mathcal{T}}(P+D) \subseteq R
$$

Thus in any case $P+\left(\cap_{D \in \mathcal{T}} D\right)=\cap_{D \in \mathcal{T}}(P+D)$. By Corollary 3.2, the $R$-module $U$ is $\lambda$-complete.

Theorem 4.2. The following statements are equivalent for a ring $R$.

(i) Every semisimple $R$-module is $\lambda$-complete.

(ii) Every simple $R$-module is $\lambda$-complete.

(iii) The ring $R$ is semiperfect.

Proof. (i) $\Rightarrow$ (ii) Clear.

(ii) $\Rightarrow$ (iii) By Lemma 4.1 and [10, Theorem 42.6].

(iii) $\Rightarrow$ (i) By Lemma 4.1 and [10, Theorem 42.6] every simple $R$-module is $\lambda$-complete and by Lemma 3.3 every semisimple $R$-module is $\lambda$-complete.

Next we investigate rings $R$ with the property that every cyclic $R$-module is $\lambda$-complete. First we recall a result of Stephenson (see [9, Theorem 1.6]).

Lemma 4.3. The following statements are equivalent for a module $M$ over a ring $R$.

(i) The lattice $\mathcal{L}\left({ }_{R} M\right)$ is distributive (i.e. $L \cap(K+N)=(L \cap K)+(L \cap N)$ for all submodules $K, L, N$ of $M)$.

(ii) $K+(L \cap N)=(K+L) \cap(K+N)$ for all submodules $K, L, N$ of $M$.

(iii) $R=\left(R x:_{R} R y\right)+\left(R y:_{R} R x\right)$ for all $x, y \in M$.

Corollary 4.4. The following statements are equivalent for a module $M$ over a ring $R$.

(i) The lattice $\mathcal{L}\left({ }_{R} M\right)$ is distributive.

(ii) Every finitely generated submodule of $M$ is a $\mu$-module.

(iii) Every 2-generated submodule of $M$ is a $\mu$-module. 
(iv) $R=\left(N:_{R} L\right)+\left(L:_{R} N\right)$ for all finitely generated submodules $N$ and $L$ of $M$.

(v) Every finitely generated submodule of $M$ is a multiplication module.

Proof. By Lemma 4.3 and [7, Corollary 3.9].

The next result is [7, Lemma 2.1].

Lemma 4.5. An $R$-module $M$ is a $\lambda$-module if and only if $(B \cap C) M=$ $B M \cap C M$ for all (finitely generated) ideals $B$ and $C$ of $R$.

We can now generalize [7, Theorems 2.3 and 3.13]. Recall that a ring $R$ is called a chain ring in case the ideals of $R$ form a chain, that is, for any ideals $B$ and $C$ of $R$ either $B \subseteq C$ or $C \subseteq B$. For any ring $R$ and prime ideal $P$ of $R$ the localization of $R$ at $P$ will be denoted by $R_{P}$ as usual. (See [6, Chapter 5] for a good account of localization.) In 1949 Fuchs [3] called a ring $R$ arithmetical provided the lattice $\mathcal{L}(R)$ is distributive and Jensen [4, Lemma 1] showed that a ring $R$ is arithmetical if and only if the local ring $R_{P}$ is a chain ring for every prime ideal $P$ of $R$.

Theorem 4.6. The following statements are equivalent for a ring $R$.

(i) $R$ is an arithmetical ring.

(ii) Every $R$-module is a $\lambda$-module.

(iii) Every homomorphic image of a $\lambda$-module is a $\lambda$-module.

(iv) Every cyclic $R$-module is a $\lambda$-module.

(v) Every finitely generated ideal of $R$ is a multiplication $R$-module.

(vi) Every finitely generated ideal of $R$ is a $\mu$-module over the ring $R$.

Proof. (i) $\Rightarrow$ (ii) Let $B$ and $C$ be any finitely generated ideals of $R$. By Corollary $4.4, R=\left(B:_{R} C\right)+\left(C:_{R} B\right)$. Then

$$
\begin{gathered}
B M \cap C M=\left[\left(B:_{R} C\right)+\left(C:_{R} B\right)\right](B M \cap C M) \\
\subseteq\left(B:_{R} C\right) C M+\left(C:_{R} B\right) B M \subseteq(B \cap C) M .
\end{gathered}
$$

It follows that $B M \cap C M=(B \cap C) M$. By Lemma 4.5 the $R$-module $M$ is a $\lambda$-module.

(ii) $\Rightarrow$ (iii) Clear.

(iii) $\Rightarrow$ (iv) Because ${ }_{R} R$ is a $\lambda$-module.

(iv) $\Rightarrow$ (i) Let $A, B$ and $C$ be any ideals of $R$. Then the cyclic $R$-module $R / A$ being a $\lambda$-module implies, by Lemma $4.5,(B \cap C)(R / A)=(B(R / A)) \cap(C(R / A))$ and hence $((B \cap C)+A) / A=((B+A) / A) \cap((C+A / A)$. It follows that $(A+B) \cap(A+C)=$ $A+(B \cap C)$. By Lemma $4.3, R$ is an arithmetical ring. 
(i) $\Leftrightarrow$ (v) $\Leftrightarrow$ (vi) By Corollary 4.4.

Theorem 4.6 applies to Prüfer domains because every finitely generated ideal is invertible and hence a multiplication module. More generally, if $R$ is a semihereditary ring (that is, every finitely generated ideal of $R$ is a projective $R$-module), then every finitely generated ideal of $R$ is a multiplication module by [8, Theorem 1 ] and hence Theorem 4.6 applies to $R$. It also applies to von Neumann regular rings because every ideal of such a ring is generated by idempotent elements and hence is a multiplication module (see [2, Corollary 1.3]).

Corollary 4.7. The following statements are equivalent for a ring $R$.

(i) Every cyclic $R$-module is $\lambda$-complete.

(ii) The ring $R=R_{1} \oplus \cdots \oplus R_{n}$ is the direct sum of chain rings $R_{i}(1 \leq i \leq n)$ for some positive integer $n$.

Proof. (i) $\Rightarrow$ (ii) By Theorem 4.2 and [1, Theorem 27.6], the ring $R=R_{1} \oplus \cdots \oplus R_{n}$ is the direct sum of local rings $R_{i}(1 \leq i \leq n)$ for some positive integer $n$. By Theorem 4.6 and [4, Lemma 1$], R_{i}$ is a chain ring for all $1 \leq i \leq n$.

(ii) $\Rightarrow$ (i) Without loss of generality we can suppose that $R$ is a chain ring. Let $A$ be any ideal of the chain ring $R$ and let $\mathcal{S}$ be any non-empty collection of ideals of $R$. Then $A \subseteq \cap_{B \in \mathcal{S}} B$ or $\cap_{B \in \mathcal{S}} B \subseteq A$. Suppose first that $A \subseteq \cap_{B \in \mathcal{S}} B$. Then

$$
A+\left(\cap_{B \in \mathcal{S}} B\right)=\cap_{B \in \mathcal{S}} B=\cap_{B \in \mathcal{S}}(A+B) .
$$

Now suppose that $\cap_{B \in \mathcal{S}} B \subset A$. Then there exists an ideal $C$ in $\mathcal{S}$ such that $A \nsubseteq C$ and hence $C \subseteq A$ because $R$ is a chain ring. In this case, it is easy to see that

$$
A+\left(\cap_{B \in \mathcal{S}} B\right)=A=\cap_{B \in \mathcal{S}}(A+B) .
$$

In any case, we have proved that $A+\left(\cap_{B \in \mathcal{S}} B\right)=\cap_{B \in \mathcal{S}}(A+B)$. By Corollary 3.2 every cyclic $R$-module is $\lambda$-complete, as required.

Now we consider finitely generated modules and ask the question: Which rings $R$ have the property that every finitely generated module is $\lambda$-complete? Are these precisely the rings for which every cyclic module is $\lambda$-complete? This amounts to asking whether chain rings $R$ have the property that every finitely generated $R$-module is $\lambda$-complete. Some chain rings do have this property. Contrast the following result with Theorem 4.6.

Theorem 4.8. Let $R$ be a local principal ideal domain. Then $R$ is a chain ring such that every finitely generated $R$-module is $\lambda$-complete but no non-zero injective $R$-module is $\lambda$-complete. 
Proof. It is well known that if $P$ is the unique maximal ideal of $R$ then the only ideals of $R$ are the ideals $R, P^{n}(n \geq 1)$ and $0=\cap_{n \geq 1} P^{n}$. Thus $R$ is a chain ring. Let $M$ be any finitely generated $R$-module. Then $M$ is a finite direct sum of cyclic $R$-modules (see, for example, [6, Theorem 10.30]) and hence $M$ is $\lambda$-complete by Theorem 4.7 and Lemma 3.3. Now let $X$ be any non-zero injective $R$-module. By [5, Proposition 2.6] and [6, Corollary 8.27],

$$
\cap_{n \geq 1}\left(P^{n} X\right)=X \neq 0=\left(\cap_{n \geq 1} P^{n}\right) X
$$

Thus $X$ is not $\lambda$-complete by Lemma 3.1 .

Finally in this section we consider rings $R$ with the property that every $R$-module is $\lambda$-complete. Note first the following simple fact which can be contrasted with Corollary 2.3.

Proposition 4.9. The following statements are equivalent for a ring $R$.

(i) Every $R$-module is $\lambda$-complete.

(ii) Every homomorphic image of every $\lambda$-complete module is $\lambda$-complete.

Proof. (i) $\Rightarrow$ (ii) Clear.

(ii) $\Rightarrow$ (i) Let $M$ be any $R$-module. There exist a free $R$-module $F$ and a submodule $K$ of $F$ such that $M \cong F / K$. By Corollary 3.4 the module $F$ is $\lambda$ complete and hence so too is $M$.

In the case of Noetherian rings we can give a complete classification. We shall require the following two lemmas.

Lemma 4.10. Let $R$ be a ring such that every $R$-module is $\lambda$-complete and let $A$ be any ideal of $R$. Then every $(R / A)$-module is $\lambda$-complete.

Proof. Let $\mathcal{S}$ be any non-empty collection of ideals of the ring $R / A$. Then every ideal of $\mathcal{S}$ has the form $B / A$ for some ideal $B$ of $R$. Let $\mathcal{S}^{\prime}$ denote the collection of ideals $B$ of $R$ such that $B / A$ belongs to $\mathcal{S}$. Let $M$ be any $(R / A)$-module. Then $M$ is an $R$-module in the usual way and we have

$$
\begin{gathered}
\left(\cap_{C \in \mathcal{S}} C\right) M=\left(\cap_{B \in \mathcal{S}^{\prime}}(B / A)\right) M=\left(\left(\cap_{B \in \mathcal{S}^{\prime}} B\right) / A\right) M=\left(\cap_{B \in \mathcal{S}^{\prime}} B\right) M \\
=\cap_{B \in \mathcal{S}^{\prime}}(B M)=\cap_{B \in \mathcal{S}^{\prime}}((B / A) M)=\cap_{C \in \mathcal{S}}(C M) .
\end{gathered}
$$

By Lemma 3.1, the $(R / A)$-module $M$ is $\lambda$-complete.

Lemma 4.11. The following statements are equivalent for a domain $R$ with field of fractions $F$. 
(i) $R$ is a field.

(ii) Every $R$-module is $\lambda$-complete.

(iii) The $R$-module $F$ is $\lambda$-complete.

Proof. (i) $\Rightarrow$ (ii) $\Rightarrow$ (iii) Clear by Lemma 3.1.

(iii) $\Rightarrow$ (i) Let $B_{i}(i \in I)$ denote the collection of all non-zero ideals of $R$. Then Lemma 3.1 gives that

$$
F=\cap_{i \in I}\left(B_{i} F\right)=\left(\cap_{i \in I} B_{i}\right) F .
$$

Thus $\cap_{i \in I} B_{i} \neq 0$. It follows that $R$ has non-zero socle and hence $R=F$.

Contrast the following result with Theorem 4.8 .

Theorem 4.12. A Noetherian ring $R$ has the property that every $R$-module is $\lambda$-complete if and only if $R$ is an Artinian principal ideal ring.

Proof. Suppose first that every $R$-module is $\lambda$-complete. Let $P$ be any prime ideal of $R$. By Lemma 4.10, every $(R / P)$-module is $\lambda$-complete and hence the domain $R / P$ is a field by Lemma 4.11. Thus every prime ideal of $R$ is maximal. By [5, Theorem 4.6], the ring $R$ is Artinian. Next, by Theorem 4.6 every ideal of $R$ is a multiplication module and hence, by [2, Corollary 2.9] every ideal of $R$ is principal. Thus $R$ is a principal ideal ring.

Conversely, suppose that $R$ is an Artinian principal ideal ring. Let $M$ be any $R$-module. Let $\mathcal{S}$ be any non-empty collection of ideals of $R$. Because $R$ is Artinian, there exists a finite subset $\mathcal{S}^{\prime}$ of $\mathcal{S}$ such that $\cap_{B \in \mathcal{S}} B=\cap_{B \in \mathcal{S}^{\prime}} B$. Noting that $R$ is a principal ideal ring and so every ideal of $R$ is a multiplication module, Theorem 4.6 and [7, Lemma 2.1] together give that $\left(\cap_{B \in \mathcal{S}^{\prime}} B\right) M=\cap_{B \in \mathcal{S}^{\prime}}(B M)$. Thus,

$$
\cap_{B \in \mathcal{S}}(B M) \subseteq \cap_{B \in \mathcal{S}^{\prime}}(B M)=\left(\cap_{B \in \mathcal{S}^{\prime}} B\right) M=\left(\cap_{B \in \mathcal{S}} B\right) M
$$

and hence $\left(\cap_{B \in \mathcal{S}} B\right) M=\cap_{B \in \mathcal{S}}(B M)$. By Lemma 3.1 the $R$-module $M$ is $\lambda$ complete.

\section{Other homomorphisms}

In general there will be many complete homomorphisms $\nu: \mathcal{L}(R) \rightarrow \mathcal{L}\left({ }_{R} M\right)$ for a given ring $R$ and $R$-module $M$ (see [7, Section 5]). Note the following result.

Proposition 5.1. Let $R$ be a ring and let $M$ be an $R$-module such that there exists a complete isomorphism $\nu: \mathcal{L}(R) \rightarrow \mathcal{L}\left({ }_{R} M\right)$. Then $M$ is a finitely generated $R$-module. 
Proof. By Lemma 1.2 because $M$ is a finitely generated $R$-module if and only if $M$ is a compact element of $\mathcal{L}\left({ }_{R} M\right)$.

Recall that a ring $R$ is called semilocal provided it contains only a finite number of maximal ideals.

Corollary 5.2. Let $R$ be a ring and let $M$ be an $R$-module such that there exists a complete isomorphism $\nu: \mathcal{L}(R) \rightarrow \mathcal{L}\left({ }_{R} M\right)$. Suppose further that either

(a) $R$ is a local ring, or

(b) $R$ is a semilocal ring and $M$ is a faithful $R$-module.

Then $M$ is a cyclic $R$-module.

Proof. By Proposition 5.1 and [7, Theorem 5.3].

Acknowledgment. The author would like to thank the referee for various helpful suggestions and in particular for bringing references [3] and [4] to his attention.

\section{References}

[1] F. W. Anderson and K. R. Fuller, Rings and Categories of Modules, SpringerVerlag, New York, 1974.

[2] Z. A. El-Bast and P. F. Smith, Multiplication modules, Comm. Algebra, 16(4) (1988), 755-779.

[3] L. Fuchs, Über die Ideale arithmetischer Ringe, Comment. Math. Helv., 23 (1949), 334-341.

[4] C. Jenson, A remark on arithmetical rings, Proc. Amer. Math. Soc., 15 (1964), 951-954.

[5] D. W. Sharpe and P. Vamos, Injective Modules, Cambridge Tracts in Mathematics and Mathematical Physics 62, Cambridge Univ. Press, Cambridge, 1972.

[6] R. Y. Sharp, Steps in Commutative Algebra, London Math. Soc. Student Texts 19, Cambridge Univ. Press, Cambridge, 1990.

[7] P. F. Smith, Mappings between module lattices, Int. Electron. J. Algebra, 15 (2014), 173-195.

[8] W. W. Smith, Projective ideals of finite type, Canad. J. Math. 21 (1969), 10571061.

[9] W. Stephenson, Modules whose lattice of submodules is distributive, Proc. London Math. Soc., 28(3) (1974), 291-310.

[10] R. Wisbauer, Foundations of Module and Ring Theory, Gordon and Breach, Philadelphia, 1991. 
Patrick F. Smith

Department of Mathematics

University of Glasgow

Glasgow G12 8QW

Scotland UK

e-mail: Patrick.Smith@glasgow.ac.uk 